\title{
Herausgeber:
}

Prof. Dr. Klaus Boers, Universität Münster | Prof. Dr. Jochen Bung, Universität Passau | Prof. Dr. Heinz Cornel, Alice Salomon-FH Berlin | Prof. Dr. Frieder Dünkel, Universität Greifswald | Prof. Dr. Andreas Eicker, Universität Luzern | Prof. Dr. Monika Frommel, Universität Kiel | Jun.-Prof. Dr. Katrin Höffler, Universität Tübingen | Prof. Dr. Johannes Kaspar, Universität Augsburg | Prof. Gabriele Kawamura-Reindl, Georg-Simon-Ohm-FH Nürnberg | Prof. Dr. Joachim Kersten, Deutsche Hochschule der Polizei, Münster | Prof. Dr. Jörg Kinzig, Universität Tübingen | PD Dr. Reinhard Kreissl, Institut für Rechtsund Kriminalsoziologie, Wien | Prof. Dr. Frank Neubacher, Universität Köln | Prof. Dr. Heribert Ostendorf, Universität Kiel | Prof. Dr. Hendrik Schneider, Universität Leipzig | Prof. Dr. Tobias Singelnstein, Freie Universität Berlin | Prof. Dr. Torsten Verrel, Universität Bonn

\section{Perspektiven für den Opferschutz im Strafverfahren}

\author{
Prof. Dr. Reinhard Böttcher, Präsident des Oberlandesgerichts a.D.
}

\begin{abstract}
Man muss heutzutage keine langen Ausführungen dazu machen, welche Folgen es haben kann, Opfer einer Straftat zu werden. Vielen ist gegenwärtig, dass Opfer etwa eines Gewalt- oder eines Sexualdelikts neben den körperlichen Folgen in vielen Fällen schwere seelische Verletzungen davontragen und oft nur mit therapeutischer Hilfe in ein halbwegs normales Leben zurückfinden. Man weiß auch, dass seelische Verletzungen nicht nur bei Gewalt- und Sexualdelikten zu erwarten sind. Sog. Hassdelikte ${ }^{1}$ z. B. können, auch wenn es nur Eigentumsdelikte sind, weit über die unmittelbaren Tatfolgen hinaus Angst und Verunsicherung erzeugen. Opfer eines Raubüberfalls, eines Wohnungseinbruchs oder einer Nachstellung (Stalking) sind als Folge der Tat vielfach seelisch geschädigt, leiden unter Angstzuständen, verlieren Vertrauen in die Umwelt, ziehen sich zurück und geraten in soziale Isolation.
\end{abstract}

Ebenso klar ist, dass nicht jedes Opfer einer Straftat über die unmittelbaren Tatfolgen hinaus tiefer gehende oder dauerhafte Verletzungen erfährt. Aber es sind doch jährlich viele, viele tausend Fälle bei uns in Deutschland, in denen das Opfer einer Straftat durch die Tat seelisch schwer geschädigt wird. Um nur eine Zahl zu nennen: Ein Delikt, bei dem eine ernsthafte seelische Schädigung geradezu typisch ist, ist der sexuelle Missbrauch von Kindern (\$S 176176b StGB). Die Polizeiliche Kriminalstatistik hat im Jahr 2011 in Deutschland etwa 13.000 Opfer des sexuellen Missbrauchs von Kindern gezählt. Dazu kommt ein Dunkelfeld, das nach allen Schätzungen erheblich größer ist als das Hellfeld.

\section{Belastung des Opfers im Strafverfahren}

Ist von Opferschutz die Rede, meint man oft die Aufgabe, Menschen davor zu bewahren, Opfer zu werden, also den präventiven Opferschutz. In diesem Sinn spricht man davon, dass der beste Opferschutz die Verhütung von Straftaten ist. Meist versteht man unter
Opferschutz aber die Aufgabe, Menschen, die Opfer einer Straftat geworden sind, vor weiterem Leid, vor zusätzlichen Verletzungen, vor einer Verlängerung und Vertiefung des Erlittenen zu bewahren und ihnen in der Situation, in die sie geraten sind, zu helfen. Opferschutz im Strafverfahren bedeutet dann, das Opfer davor zu schützen, dass es durch das Strafverfahren zusätzlich verletzt wird und es darin zu unterstützen, dass es sich in dem Verfahren behaupten kann. Davon soll im Folgenden die Rede sein.

Es geht um Menschen, die Opfer einer Straftat geworden sind, nicht um solche, die das fälschlich behaupten. Auch die gibt es, wenn auch verhältnismäßig selten. Solche Fälle aufzudecken, das angebliche Opfer zu entlarven, ist nicht nur ein selbstverständliches Anliegen des jeweils Beschuldigten und ein Anliegen des Rechtsstaats, sondern auch im Interesse des Opferschutzes. Jede fälschliche Inanspruchnahme der Opferrolle fügt dem Opferschutz Schaden zu. Opferschutz wendet sich nicht gegen die Feststellung des wahren Sachverhalts, im Gegenteil. Opferschutz akzeptiert auch den Rechtssatz in dubio pro reo, auch wenn das für das betroffene Opfer schmerzhaft sein kann.

Dass das Strafverfahren für das Opfer gefahrenträchtig ist, wird heute weithin anerkannt. Die Gefahren sind vielfältig und es hängt von unterschiedlichen Faktoren ab, ob und wovor ein bestimmtes Opfer sich fürchten muss. Sicher eine besonders furchterregende Situation droht dem Opfer im Fall eines schambesetzten Delikts im sozialen Nahbereich, etwa einer Vergewaltigung oder eines Missbrauchs, das vom Täter bestritten wird. In einem solchen Fall muss das Opfer fürchten, dass das Verfahren zur Schlacht um seine Glaubwürdigkeit und moralische Integrität wird. Haftet dem Fall dann noch irgend etwas Spektakuläres an, das das Interesse der Medien aufstachelt, so kann das Opfer das Gefühl haben, dass es nicht so sehr um die Zukunft des Täters als vielmehr um seine, des Opfers, moralische 
und soziale Zukunft gehen wird, und das auch dann, wenn alle Beteiligten, auch die Verteidiger, auch die Medien, sich gegenüber dem Opfer korrekt verhalten werden. Und wer kann das garantieren?

Es sind nicht nur solchermaßen zugespitzte Konstellationen, die dem Opfer Angst machen. Auch wenn der Täter gesteht oder durch Spuren eindeutig überführt wird, hat das Opfer Gründe, sich vor dem Verfahren zu ängstigen. Dass es im Verfahren als Zeuge aussagen muss, in der Regel mehrfach, und dabei das Geschehen in ihm wieder hochkommt, dass es dem Täter vor Gericht begegnen kann, dass das Umfeld des Opfers von der Sache erfährt und vielleicht auch die örtliche Zeitung berichtet, das alles kann dem Opfer Angst machen. Anderer Art sind die Belastungen, wenn der Täter oder sein Umfeld versucht, Einfluss auf die Zeugenaussage des Opfers zu nehmen, das gibt es nicht nur in Fällen aus dem Bereich der organisierten Kriminalität, oder wenn die Medien hinter dem Opfer her sind.

Opfer sind im Strafprozess gegen den Verdächtigen in vielen Fällen Belastungen ausgesetzt, für die man erst einmal eine Rechtfertigung finden muss. Wir sagen dann, es geht um die Durchsetzung des Rechts, es geht um Normstabilisierung und Prävention im Allgemeininteresse. Dafür wird das Opfer in Anspruch genommen. Das Opfer muss ein Sonderopfer für die Allgemeinheit erbringen. Das schreit geradezu nach Unterstützung und Hilfe.

\section{Die Anzeige als soziale Leistung}

Dem weit überwiegenden Teil der Ermittlungs- und Strafverfahren liegt eine Anzeige des Opfers zugrunde, nur verhältnismäßig wenige Straftaten werden der Polizei auf andere Weise bekannt. Es ist deshalb ein für die Strafrechtspflege bedeutsamer Vorgang, wenn ein Opfer nach beendetem Strafverfahren gegen den Täter erklärt, nie mehr würde es eine Anzeige erstatten. Das kommt leider immer wieder vor. Es ist nur der kleinere Teil der begangenen Straftaten, der zur Kenntnis der Behörden kommt, der Rest verbleibt im Dunkelfeld. Wenn die Anzeigebereitschaft zurückgeht, wächst das Dunkelfeld zu Lasten des Hellfeldes noch weiter, ein bedenklicher Vorgang in Bezug auf die Durchsetzung des Rechts. Andererseits: Hält man sich vor Augen, was auf das Opfer nach Erstattung einer Anzeige alles zukommen kann, ist es verwunderlich, dass viele von ihnen nicht den Mut aufbringen, Anzeige zu erstatten?

Dazu, welche Gründe Opfer zu einer Anzeige motivieren, gibt es Befragungen, aber auch viel Erfahrungswissen. Neben der Schwere des Delikts (schwere Taten werden eher angezeigt als leichte) und etwa einschlägigen Versicherungsbedingungen, die auf einer Anzeige als Voraussetzung der Schadensregulierung bestehen oder nicht bestehen, spielen v. a. zwei Motive eine Rolle: Der Wunsch, dass Gerechtigkeit hergestellt wird, die Tat nicht ungesühnt bleibt, und das Anliegen, zu verhindern, dass anderen Menschen Gleiches widerfährt. Das sind Motive, die großen Respekt verdienen. Entgegen einer häufig gehörten Unterstellung geht es Opfern meist nicht um Rache, dazu gibt es schöne Aussagen von Richard Oetker und Jan Philipp Reemtsma, beide Opfer schwerster Verbrechen, sondern darum, dass der Staat, die Rechtsgemeinschaft, das dem Opfer angetane Unrecht als solches anerkennt und den Täter in die Verantwortung nimmt. Wenn Opfer sich in Fällen, in denen das Verfahren absehbar mit großen Belastungen für sie verbunden ist, aus solchen Motiven zu einer Anzeige entschließen, erbringen sie für den Rechtsstaat eine Leistung, die Anerkennung verdient. Diese Leistung macht es zusätzlich dringlich, die Belastungen für das Opfer so gering wie möglich zu halten und ihm Gelegenheit zu geben, an der Feststellung der Wahrheit und dem Finden eines gerechten Urteils mitzuwirken.

\section{Opferschutz als Thema}

Es hat lange gedauert, bis sich diese Erkenntnis durchgesetzt hat. Lange Zeit hat die Politik, hat aber auch die Wissenschaft mehr oder weniger unkritisch hingenommen, dass das Opfer im Strafverfahren im Wesentlichen ein Beweismittel ist, sich als Mittel zur Ermittlung des wahren Sachverhalts zur Verfügung zu stellen hat, und weiter nichts. Das galt nicht ausnahmslos. In Einzelbereichen gab es Möglichkeiten aktiver Mitwirkung für das Opfer, im Ganzen gesehen aber war es so, wie Rieß 1984 geschrieben hat: In einer langen Entwicklung hatte das Straf- und Strafverfahrensrecht den Verletzten in eine Randposition abgedrängt ${ }^{2}$. Oder, wie es später bei Kilchling hieß: Das Opfer war zu einer „prozessualen Restgröße“ degradiert worden $^{3}$. Erst Ende der siebziger Jahre, 1976 war der WEISSE RING gegründet worden, begann in Deutschland eine kritische Diskussion der Opferrolle im Strafprozess, die dann in den Beratungen des 55. Deutschen Juristentags 1984 im Grundsätzlichen wie in vielen Einzelforderungen überzeugenden Ausdruck fand. Nahezu einstimmig forderten die damals in der strafrechtlichen Abteilung des Juristentags versammelten Juristen, den Verletzten besser davor zu schützen, dass er durch das Strafverfahren zusätzlich beeinträchtigt wird, und ihn besser in Stand zu setzen, sich gegen Verantwortungszuweisungen und Diskriminierungen zu verteidigen. Mehrheitlich forderte man außerdem, dem Verletzten bessere Möglichkeiten zum Ausgleich des ihm entstandenen materiellen und immateriellen Schadens zu schaffen. Aus diesen Grundsätzen hat der Juristentag viele konkrete Vorschläge abgeleitet und eine konsensfähige Plattform für die danach alsbald, nämlich 1985, eingeleitete Reformgesetzgebung geschaffen.

Warum hat es so lange gedauert, bis die Belastungen des Opfers durch das Strafverfahren, seine Leistung für die Strafrechtspflege zum Thema wurden? Lag es nicht immer schon nahe, sich nach einem Verbrechen zunächst einmal anteilnehmend mit dem Opfer zu beschäftigen?

Vielleicht stand dem lange Zeit entgegen, dass man die Straftat vor allem als Auflehnung gegen das staatliche Gebot, weniger als Verletzung der Rechtsgüter eines Mitbürgers gesehen hat. Der Strafprozess war eine Veranstaltung zwischen dem Staat und dem Beschuldigten, bei der man dem Angeklagten ein faires Verfahren sichern musste. Für das Opfer blieb keine Aufmerksamkeit. In späteren Jahren war man teilweise darauf fixiert, mit dem Strafverfahren die Resozialisierung des Täters zu bewirken. Für eine eigenständige Rolle des Opfers war auch da kein Platz. ${ }^{4}$ Jedenfalls hat es bis in die achtziger Jahre gedauert, bis Opferschutz im Strafverfahren in Deutschland ein Thema für die Politik wurde.

\section{Opferschutzgesetzgebung}

Dann allerdings ging es Schlag auf Schlag. Beginnend mit dem Opferschutzgesetz von $1986^{5}$, fortgeführt mit dem Zeugenschutzgesetz von $1998^{6}$, dem Gewaltschutzgesetz von $2001^{7}$ und dem Opferrechtsreformgesetz von $2004^{8}$ und vorläufig endend mit dem 2. Opferrechtsreformgesetz von $2009^{9}$, um nur die größeren Reformgesetze zu nennen, hat der Bundesgesetzgeber die Rechtsposition des Opfers im Strafprozess in wesentlichen Punkten gestärkt.

Das Recht des Opfers auf Erhalt von Informationen wurde ausgeweitet, Informationen zum Gang des Verfahrens, Informationen 
über die Rechte des Opfers und Informationen über bestehende Möglichkeiten der Unterstützung und der Hilfe. Das Opfer erhielt das Recht, sich grundsätzlich bei allen Vernehmungen durch eine Person seines Vertrauens begleiten zu lassen. Das Recht des Opfers, sich durch einen Rechtsanwalt begleiten und unterstützen zu lassen und mit seiner Hilfe Einsicht in die Verfahrensakten zu nehmen, wurde geregelt. Erheblich ausgeweitet wurde das Recht des Opfers, sich, unterstützt durch einen Rechtsanwalt, aktiv am Verfahren zu beteiligen. Besonders schutzbedürftige Gruppen von Opfern erhalten zu diesem Zweck kostenlos einen Rechtsanwalt beigeordnet. Der Schutz des Opfers vor diskriminierenden Fragen und vor einer bedrohlichen Konfrontation mit dem Angeklagten wurde verstärkt. Durch Einsatz der Videotechnik bei der Vernehmung kann jetzt eine Konfrontation mit dem Angeklagten in bestimmten Fällen vermieden werden, sei es, dass das Opfer aus der Hauptverhandlung heraus per Video vernommen wird, sei es, dass eine vor der Hauptverhandlung vorgenommene und aufgezeichnete Vernehmung eingespielt wird. Der Schutz vor öffentlicher Bloßstellung wurde verbessert. Die Möglichkeit, zum Schutz der Intimsphäre die Öffentlichkeit von der Hauptverhandlung auszuschließen, wurde erweitert. Das Recht des Opfers, Schmerzensgeld und Schadensersatz schon im Strafverfahren geltend zu machen, wurde erweitert. Das Gewaltschutzgesetz hat auf zivilrechtlichem Wege die Position des Opfers gestärkt. Das Gericht kann Schutzanordnungen erlassen, deren Nichtbeachtung strafbar ist. Bei einer gemeinsam genutzten Wohnung gilt der Grundsatz:"Wer schlägt, der geht".

Es ist eine beeindruckende Zahl opferschützender Entscheidungen, die der Gesetzgeber getroffen hat. Es gibt keinen Aspekt des Strafverfahrens, dem er in den vergangenen 25 Jahren so viel Aufmerksamkeit geschenkt hat wie dem Opferschutz. In der Wissenschaft wird deshalb darüber diskutiert, ob man in Bezug auf den Strafprozess von einem Paradigmenwechsel sprechen kann. Keine Frage ist, dass der Gesetzgeber ein lange unbeachtetes Thema mit Nachdruck aufgegriffen und dem Strafprozess in wiederholten Anläufen mit der Einbeziehung der Opferinteressen eine zusätzliche Dimension gegeben hat.

Dabei geht die Entwicklung im Augenblick weiter. Aus den Beratungen des von der Bundesregierung im Jahr 2010 eingesetzten Runden Tisches, der über Folgerungen aus den bekannt gewordenen Fällen sexuellen Kindesmissbrauchs in Institutionen und im familiären Bereich beraten hat, ist der Regierungsentwurf eines Gesetzes zur Stärkung der Rechte von Opfern sexuellen Missbrauchs ${ }^{10}$ hervorgegangen, der derzeit im Rechtsausschuss des Bundestags beraten wird. Neben einer Verlängerung der Verjährungsfrist für einschlägige Straftaten soll, ohne strenge Begrenzung auf die Fälle sexuellen Missbrauchs, der Schutz von Opfern im Strafverfahren weiter verbessert werden. So sollen Mehrfachvernehmungen weitergehend zurückgedrängt werden. Opferzeugen sollen ungeachtet der Beweislage ein Recht zur Äußerung zu den Tatfolgen haben; das sog. victim impact statement, dem international große Bedeutung zugemessen wird $^{11}$, soll also Eingang in das deutsche Recht finden. Die Regelung über den staatlich bezahlten Opferanwalt erfährt eine kleine Ausweitung. Die Information des Opfers über Vollzugslockerungen für den Täter wird verbessert. Der Schutz jugendlicher Opfer vor der Öffentlichkeit wird um eine Nuance gestärkt. Man sieht: Unter verschiedenen Aspekten soll der Opferschutz noch einmal verbessert werden. Zusammen gesehen ist es ein recht ansehnliches Paket an Vorschlägen, das am Runden Tisch erarbeitet und dem Gesetzgeber zur Verabschiedung empfohlen worden ist, praktisch einmütig übrigens, ungeachtet der am Runden Tisch vertretenen unterschiedlichen
Positionen. Alle standen unter dem Eindruck, dass viele, viele Missbrauchsopfer über Jahre und Jahrzehnte geschwiegen haben und sahen eine der notwendigen Schlussfolgerungen darin, Opfern die Entscheidung für eine Anzeige zu erleichtern, indem man die Belastungen, die im Strafverfahren auf sie warten, verringert.

\section{Ende der Fahnenstange?}

Weniger das letztgenannte Gesetzesvorhaben, aber die vorgangegangenen Opferschutzgesetze, insbesondere das Zweite Opferrechtsreformgesetz, haben neben Lob auch Kritik gefunden, nicht in der Politik, aber in der Rechtswissenschaft. Sie äußerte sich in literarischen Stellungnahmen ${ }^{12}$, bei wissenschaftlichen Tagungen ${ }^{13}$, fand aber auch einen scharfsinnigen Vertreter bei der Anhörung, die der Rechtsausschuss des Bundestags zum 2. ORRG durchgeführt hat. Jahn hat damals die Frage aufgeworfen, ob die vorgesehene Stärkung der Verletztenrechte den verfassungsrechtlichen Anspruch des Beschuldigten auf ein faires Verfahren verletze. Im Ergebnis hat zwar er einen angreifbaren Verfassungsverstoß verneint, aber doch gemeint, man müsse erörtern, ob der Ausbau der Verletztenrechte seit 1986 bei gleichzeitigem Ausbleiben einer nachhaltigen Stärkung der Beschuldigtenrechte dazu geführt habe, dass eine unter Fairnessgesichtspunkten verfassungsrechtlich bedenkliche Schieflage entstanden sei.

Im Gesetzgebungsverfahren blieben diese Zweifel damals ohne Wirkung. Bundesregierung und Bundestag gingen, wie sich aus der Beschlussempfehlung des Rechtsausschusses des Bundestags ${ }^{14}$ entnehmen lässt, davon aus, dass mit dem Gesetz im Gegenteil einer verfassungsrechtlichen Verpflichtung des Staats nachgekommen wird, der Verpflichtung, sich schützend vor die Opfer von Straftaten zu stellen.

Haben sie geirrt? Hat sich durch die Opferschutzgesetzgebung die „Balance“ zwischen den Beteiligten des Strafprozesses in einer Weise verschoben, dass rechtsstaatliche Zweifel insbesondere unter dem Gesichtspunkt der Verfahrensfairness begründet sind? Die Sorge ist m. E. nicht begründet. Das Fairnessgebot darf nicht auf seine Schutzwirkung für den Beschuldigten verkürzt werden. Schon in einer frühen Entscheidung ${ }^{15}$ hat das Bundesverfassungsgericht klargestellt, dass das Fairnessgebot auch den Schutz der anderen Beteiligten bezweckt. Auch ihnen garantiert es das Recht, angemessen behandelt zu werden und Übergriffe von staatlicher Seite oder anderer Verfahrensbeteiligter abwehren zu können. Geht man aber davon aus, dass das Fairnessgebot auch das Opfer schützt, so muss, wenn fairer Opferschutz und fairer Beschuldigtenschutz zu unterschiedlichen Ergebnissen führen sollten, im Einzelfall abgewogen werden und im Wege praktischer Konkordanz ein schonender Ausgleich hergestellt werden, wie auch sonst, wenn verfassungsrechtlich geschützte Positionen kollidieren. Keinesfalls setzt das Fairnessgebot dem Opferschutz einseitig Grenzen. Die im Wege des schonenden Ausgleichs zu bestimmenden Grenzen des Opferschutzes sind noch lange nicht erreicht.

In der Praxis wird es so sein, dass die Entfaltung des Opferschutzes in der Gesetzgebung seit 1986, dass nicht zuletzt aber auch das Heranwachsen einer Anzahl engagierter Opferanwältinnen und Opferanwälten die Strafverteidigung in manchen Fällen schwieriger gemacht hat. Strafverteidigung auf dem Rücken des Opfers ist nicht mehr so leicht. Aber das ist ein Fortschritt. Niemand will dem Beschuldigten sein Recht auf effektive Verteidigung nehmen. Aber ein Recht des Beschuldigten, sich ungestört zu Lasten des Opfers zu ver- 
teidigen, ein Recht auf ein wehrloses Opfer, das gibt es nicht, wie Schöch in der erwähnten Anhörung richtig gesagt hat.

Weitere Verbesserungen der Rechtsstellung des Opfers im Strafprozess sind zulässig und sie sind wünschenswert. Der WEISSE RING hat dazu eine lange Wunschliste erarbeitet. Es sollen hier nur drei Anliegen angesprochen werden.

Zunächst geht es um die Einstellung von Verfahren wegen eines Vergehens gegen Auflagen, in der Regel eine Geldauflage, wenn die Schuld nicht zu schwer wiegt und das öffentliche Interesse nicht entgegensteht ( $\$ 153 \mathrm{a}$ StPO). Diese Verfahrensweise wird massenhaft praktiziert, hat sehr große Bedeutung. Das Opfer muss in der Regel nicht gehört werden, erst recht nicht zustimmen, und kann die Einstellung auch nicht im sog. Klageerzwingungsverfahren angreifen. Das ist Opfern in manchen Fällen schwer zu vermitteln.

Sodann geht es darum, dass das Verfahren auf einen oder mehrere Tatvorwürfe beschränkt wird und andere Tatvorwürfe aus dem Verfahren ausgeschieden werden, weil sie für die erwartete Sanktion, wie das Gesetz sagt, nicht beträchtlich ins Gewicht fallen $(\mathbb{S} 154$ StPO). Das Opfer kann sich nicht dagegen wehren, auch nicht das Opfer einer Tat, die aus dem Verfahren ausgeschieden wird, selbst wenn diese ein Verbrechen ist. Es muss zuschauen, wie das Verfahren wegen der von ihm angezeigten Tat, einer Tat, die vielleicht sein Leben nachhaltig zum Schlechten verändert hat, fallengelassen wird, weil es auf sie nach Meinung der Justiz „nicht beträchtlich“ ankommt. In der Praxis ist das für die Betroffenen in vielen Fällen ein Schock und zerstört das Vertrauen, das sie mit ihrer Anzeige in den Rechtsstaat gesetzt haben, nachhaltig.

Ein dritter Punkt ist die Beteiligung des Opfers bei der Verständigung im Strafverfahren. Zwar muss der Nebenkläger in der Hauptverhandlung dazu gehört werden. Doch kann er sich nicht dagegen wehren, wenn Gericht, Staatsanwaltschaft und Verteidigung sich aus Gründen der Verfahrensökonomie auf eine Sanktion einigen, die die Tat verharmlost, oder, noch schlimmer, die vom Opfer erlittene Tat aus dem Verfahren ausgeschieden wird. Hat sich das Opfer schweren Herzens zur Anzeige entschlossen, ist das eine frustrierende Erfahrung.

In allen drei Punkten gilt es, die Position des Opfers zu stärken. Die Strafprozessordnung ist noch längst nicht in jeder Hinsicht opferfreundlich, Opferbelange werden teilweise geradezu missachtet ${ }^{16}$.

\section{Perspektiven}

Was weiteren Fortschritten rechtspolitisch entgegensteht, ist v.a. der befürchtete Verfahrensaufwand. Man kann nicht bestreiten, dass ein weiterer Ausbau der Opferrechte, insbesondere wenn es sich um Aktivrechte handelt, das Verfahren insgesamt komplexer macht und damit zusätzlich Ressourcen auf Seiten der Strafjustiz, u. U. auch auf Seiten der Polizei, in Anspruch nehmen kann. In Zeiten von Sparhaushalten begründet das schwer zu übersteigende Barrieren.

Es wird, jedenfalls längerfristig, trotzdem zu weiteren Fortschritten beim Opferschutz im Strafprozess kommen. Für diese Prognose gibt es zwei Gründe.

Der eine Grund ist der Rückenwind aus Europa. Opferschutz ist nicht nur ein Thema in Deutschland,in anderen Staaten und in internationalen Organisationen wie den Vereinten Nationen, er ist auch ein Thema der Europäischen Union. 2001 hat der Rat den Rahmenbeschluss über die Stellung des Opfers im Strafverfahren ${ }^{17}$ erlassen, mit dem den Mitgliedstaaten eine Reihe opferfreundlicher Zielset- zungen vorgegeben wurde. In Deutschland wurden einzelne Anregungen in den Opferrechtsreformgesetzen von 2004 und 2009 aufgegriffen. In den zurückliegenden Monaten wurde in der Union über einen Vorschlag der Kommission für eine Richtlinie des Parlaments und des Rates über Mindeststandards für die Rechte und den Schutz von Opfern sowie für die Opferhilfe ${ }^{18}$ beraten, die den Rahmenbeschluss ersetzen und den Opferschutz in den Mitgliedstaaten weiter voranbringen will. Eine Einigung ist soeben erfolgt ${ }^{19}$. Die Veröffentlichung steht bevor. Es ist wahrscheinlich, dass sich auch aus dieser Richtlinie, deren Zielvorgaben für die Mitgliedstaaten verbindlich sind, Regelungsanstöße für Deutschland ergeben, jedenfalls in dem einen oder anderen Punkt.

Der andere Grund ist die Entwicklung in Deutschland in den zurückliegenden 25 Jahren. Sie ist von Anfang an von einem breiten politischen Konsens getragen worden. Ihr liegen wissenschaftliche Erkenntnisse zugrunde, die den Menschen die Augen dafür geöffnet haben, welch gravierende Folgen bis hin zu einer posttraumatischen Belastungsstörung Gewalt- und Missbrauchserfahrungen für die Opfer haben können. Es hat sich ein System der Hilfe und der Unterstützung entwickelt, das von einflussreichen karitativen Organisationen getragen und von einer wachsenden $\mathrm{Zahl}$ engagierter Opferanwälte, Opferhelfer und Therapeuten praktiziert wird. Die Sicht der Strafverfolgungsbehörden auf das Strafverfahren ist differenzierter geworden, sozusagen mehrpolig. Die Fixierung auf die Überführung und Bestrafung des Täters ist einer Betrachtung gewichen, in der auch Platz für die Opferbelange ist. Die Medien machen die Erfahrung, dass sich Interesse und Anteilnahme der Leser/Zuschauer auch mit Opferschicksalen ansprechen lässt und berichten über diese deutlich mehr als früher. Opferschutz stößt in der Bevölkerung auf breite Zustimmung.

Warum sollte die Entwicklung abbrechen oder gar rückgängig gemacht werden? Alle Beteiligten und viele Betrachter spüren, dass der Umgang mit der Kriminalität durch die Berücksichtigung der Opferbelange gewonnen hat, an Realitätsbezug und an Mitmenschlichkeit.

\section{Fußnoten:}

1 Dazu Schneider, Hass- und Vorurteilskriminalität, in: Schneider (Hrsg.) Internationales Handbuch der Kriminologie, Band 2, 2009, $297 \mathrm{ff}$

2 Rieß, Gutachten C zum 55. DJT, 1984, C 45.

3 Kilchling, Opferschutz und Strafanspruch des Staates - Ein Widerspruch?, NStZ 2002, 57 ff., 61.

4 Ähnlich Kilchling, aaO, 58 ff; Weigend, „Die Strafe für das Opfer“? - Zur Renaissance des Genugtuungsgedankens im Straf- und Strafverfahrensrecht, RW 2010, 39 ff, 40/ 41; Schneider, Viktimologie, in: Schneider (Hrsg.), Internationales Handbuch der Kriminologie, Bd. 1, 2007, S. 395 ff., 412.

5 Gesetz vom 18. 12. 1986, BGBl. I S. 2496.

6 Gesetz vom 30. 4. 1998, BGBl. I S. 820.

7 Gesetz vom 11. 12. 2001, BGBl. I S. 3513.

8 Gesetz vom 24. 6. 2004, BGBl. I S. 1354.

9 Gesetz vom 29. 7. 2009, BGBl. I S. 2280.

10 StORMG, BT-Drs. 17/6261.

11 Schneider, Neue Auswirkungen der kriminologischen Verbrechensforschung - ihre Auswirkungen auf die Opferpolitik, JR 2010, 375 ff.,384.

12 Nachweise bei Weigend, Das Opfer als Prozesspartei?, FS Schöch 2010, $947 \mathrm{ff}$.

13 Z. B. Tagung „Ambivalenzen der Opferzuwendung des Strafrechts“ unter Leitung von Barton und Kölbel am 14./15. 92011 an der Universität Bielefeld.

14 BT-Drs. 161/ 13671.

15 BVerfGE 38, 105, 122

16 Vgl. Kilchling, Opferschutz und Strafanspruch des Staates - Ein Widerspruch?, NStZ 2002, 57 ff., 60/61.

17 RB vom 15. 3. 2001, ABl. 82,1.

18 KOM (2011) 275 endgültig.

19 Vgl. Standpunkt des EP v. 12.9.2011 P7_TC1_COD (2011)0129. 